\title{
Research on the Design of Public Traffic Information System
}

\author{
Dan Zhang ${ }^{1, \text { a }}$ \\ ${ }^{1}$ 99Songling Road,Qingdao,China \\ aqdzhangdan@163.com
}

Keywords: public traffic information system design principle sign

Abstract. As a visiting scholar at Massachusetts Institute of Technology for one year, the author has done a full investigation and study on the subway lines of B-line, C-line, D-line, E-line, Red line and Orange line in Boston, the subway and bus traffic in Canada traffic information system combined with the actual project. With the successful experience of domestic and foreign traffic site design, the actual situation of China public traffic information system is analyzed and summarized, considering the relationship with the historical and the cultural characteristics of China. In the end, the basic principles of traffic information system design is introduced, which can contribute to the future construction and development of Chinese public traffic information system.

\section{Introduction}

With the improvement of economic, the rapid development of urban construction, and the rapid increase of urban population, the lag of public transport facilities and other issues have gradually revealed. As an important information carrier of urban transport facilities, the public traffic information system is a very necessary system engineering in today's China, especially through the accelerated urbanization. The traffic information system plays an irreplaceable role, it will make people travel more convenient, so that the urban environment becomes more harmonious and orderly, and the public traffic of the city will also be greatly improved.

\section{The importance of traffic information system design}

In the processing of urbanization of the major cities, more people have emerged in the city. It has been expanding the size of the population and has the rapid growth in the number of motor vehicles, traffic overload and so on. After a certain extent development, the metropolis began to adjust the transport structure, develop urban public transport, and build rail transport, which establish an integrated transport system that actively and vigorously to ease urban traffic pressure, improve the efficiency of travel, and guide the rationalization of urban layout.

With the vigorous development of urban rail transit in China, traffic construction has gradually become the focus. The traffic information system is the important carrier of information, the important medium to contact environment and people's behavior. In our busy, fast-paced life, the traffic information system plays an important role in guiding the direction, indicating the behavior, marking the nature of the place and location and so on. It is a simple, efficient, convenient, easy-to-read and secure information identification system, which can transmit information quickly and accurately and guide people's behavior by text, mark and graphic symbols. In the traffic behavior, passengers can reach the destination in the most convenient, fast, comfortable and clear circumstances.

Therefore, through the research and analysis on the projects and case study of the traffic information system design in developed countries and domestic transportation sites in the United States, Canada and other countries, we have followed the design principle of "people-oriented", which makes people realize green travel, embodying urban quality and humanistic care to meet different people, especially the disabled, women and children, the elderly and other vulnerable groups needs. So as to provide effective help and guidance for the design of traffic information system in the future. 


\section{Classification of traffic information system}

In the traffic site, according to different modes of transport, the information system can be divided into airport information system, rail and urban rail transit information system, and bus information system; according to different functions, it can also be divided into information signs, name signs, warning signs and electronic signs.

information signs: It is to provide information for people and establish the identification card. Such as: road maps, subway maps, landmarks, price lists, timetables and so on.

guiding signs: It is to guide the direction, the action line for people and establish the identification card. Such as: the signs of station entrance and exit, sidewalk signs and so on.

name signs: simple, clearly indicate the name of this place, so that people can quickly identify. Such as: subway and bus station signs and so on.

warning signs: In order to protect the safety of public, set up signs of danger away. It has the meaning of mandatory compliance generally, identifies the acts prohibited by the act and norms clearly. Such as:prohibit taking pictures, prohibited pets, prohibited to carry fire and so on.

electronic signs: With the using of modern science and technology,the electronic guide system can give people a lot of information. Such as: electronic rolling information systems, interactive electronic touch systems.

\section{Design Principle of the traffic Information System}

A successful traffic information system design can provide an enjoyable experience for people who are involved in public transport. It requires information system to be content clear and concise, and requires visual communication to be quick and accurate. The important factors and principles of the traffic information system design are discussed in the following context.

It has regional characteristics. The content, shape and color of the traffic information system are coordinated with the environment and in accordance with the local style and preferences. It reflects the regional, cultural and identifiable characteristics that can beautify the environment.

Because of the specific geographical, historical, cultural and the formation of different environmental styles in each country and city, different regions have their own characteristics, such as the Kendal subway station traffic information system of Massachusetts Institute of Technology showing the development history of MIT, which embodies the deep cultural background of this world-class institutions.

The traffic information system should be set in people's attention that can be seen quickly. Such as: road junctions, subway station, the ticket office. According to the different locations, structure and form, public traffic information system can be divided into: hanging information system, mosaic information system, wall information system, opening information system, closed information system, and stand-alone information system and so on.

It requires reasonable and ergonomic scale. As British psychologist D. Canter said: "In the past many of the incorrect design is from the rules and regulations that caused by the human wrong behavior. It can help to get rid of these fetters if you can understand the people more fully, more clearly, and more scientific. Then it can create greater architectural art works that are more satisfied with." For the size of traffic information system, it requires to follow the people-oriented, human service design concept, to better meet passengers comfortable, safe and efficient demand. Based on the data collected by anthropologists and the information collected on various representative users, including body size that are sorted by age, sex, ethnicity, etc, it should be appropriate for most people based on the general size .

It requires colorful and easy to identify. The stronger the color contrast, the easier it is to recognize by the human eye. Such as yellow on black, white on black, blue on white, and black on white, these colors can highlight the information, which play the better role in information identification system. On the other hand, the color design should also follow the rules of formal beauty, such as contrast, rhythm, and symmetrical balance. The application of these rules will make the traffic information system more in 
line with people's aesthetic requirements, thus affecting the pedestrian recognition and identification of interest, which can be identified and use pleasantly and achieve the harmonious coexistence of people and things.

The same line, function, category of traffic information system should have a unified color, so as to give people a consistent impression. For example: Boston subway is divided into red line, green line, purple line, silver line and different lines. According to the different lines, the subway information system uses the corresponding color logo information that can be identified and distinguished easily.

The text and graphics should be easy to read.

Graphical symbols and text messages are easy to identify, concise and clear, so passengers can get rapid access to information at a glance. The explanatory text is communicated in both international and local languages. The traffic sign uses the general and international nature of the graphical symbols on the identification plate, which make the convention of the symbols to accurately convey the information to all countries.

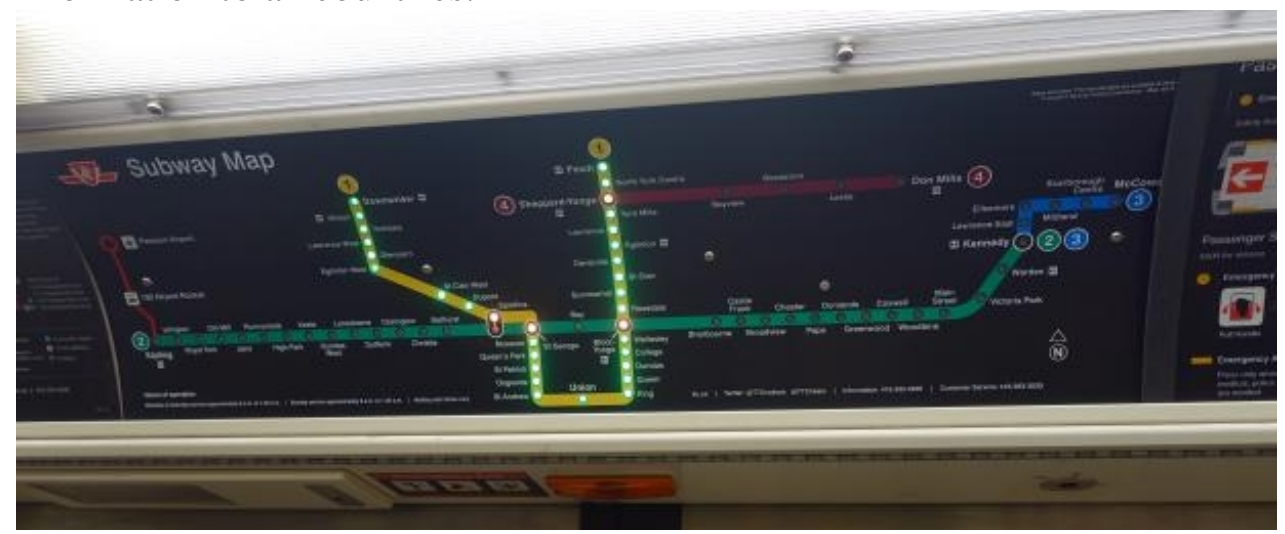

Figure1 Toronto subway map

Site information, driving maps and electronic display system are complete, orderly and easy to read. For example: Boston subway map from the starting to the terminal station uses the line expression that can be easy to read. If conditions permit, it can also use electronic display. For example: It is red flashing, more eye-catching when the train is approaching in Toronto electronic subway map. (Figure1) It should be attention to people's movement, speed and ability to identify. For example, on a fast-moving car, we can only identify the larger text on the roadside sign and a little amount of content. While the sidewalk signs we can stop to read the information. Thus, in the state of travel, it is more difficult for people to obtain information with faster speed. Therefore, in order to increase recognizable, the traffic information system would include text content, font, font size, size and other factors; it should give full consideration to people's movement state and speed.

It requires barrier-free traffic information system.

The integrated system for people with disabilities is designed for blind people, visually impaired persons or wheelchair users. It should be a significant reduction in the use of information for the normal in design. It will meet the resolution of the finger touch; and finger resolution and visual resolution ratio is 1: 1000, it makes the contours of the braille and graphics clear by touch .It can be identified in order to reflect the community's humanistic care for people with disabilities. At the same time, the designers should also pay attention to the use of the logo for the disabled, which is an international symbolic designation, it means "facilities accessible to the disabled". The figure is expressed in wheelchair form, and wheelchair users are the main users, but not the only object used.

During living in Boston, I had deep feelings in the public facilities, especially the accessibility of the traffic site design. People with physical disabilities, who rely on wheelchairs, are fully able to go out alone. It was showing that barrier-free design has spread to all aspects of life. In the subway ticket site, self-service ticketing machine engraved with braille surface to facilitate the blind ticketing; the traffic information station is marked with wheelchair signs which are barrier-free design. That means the disabled can be independent and convenient access to reach where are equipped with accessible ramp or dedicated elevator. (Figure2) 


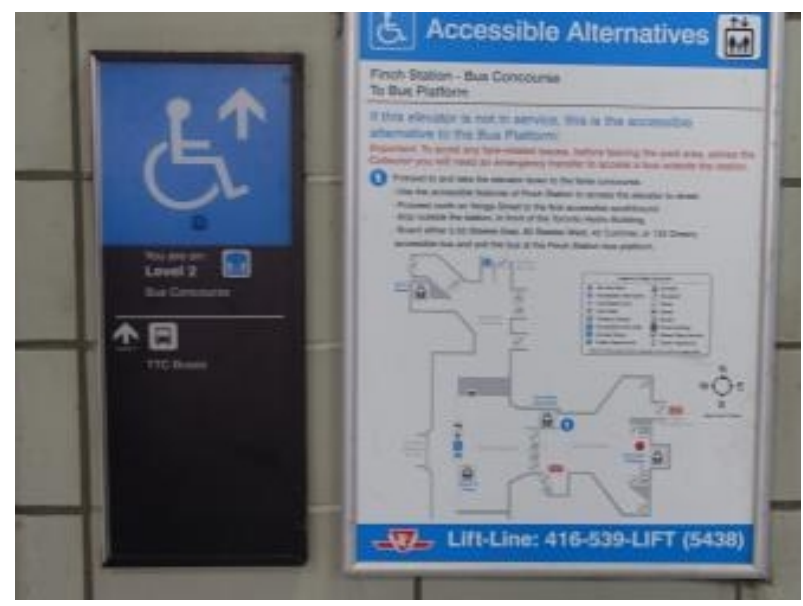

Figure2 Accessible sign

Therefore, the "people-oriented" barrier-free design has always been reflected in the modern urban traffic information system. It requires to make the passengers in the first order in design, concerning for the people anytime and everywhere, concerning about the user's psychological needs and physiological needs, and reflecting the spirit of the user care.

\section{Conclusions}

Based on the investigation and study of advanced design achievements and theories, this paper conducts a more comprehensive study on traffic information system. Through learning and drawing lessons from the outstanding projects and experiences of developed countries in Canada and America, this paper aims to provide some reference value for the design of traffic information system in the future, and to provide reference for the follow-up research in the related fields.

\section{Acknowledgments}

This work was financially supported by China Scholarship Council(No. 201507890018), the special Project of art education of Shandong Province "Research on the Importance of Cultivation of Innovative Talents in Universities" and the special project of women and traditional culture of Shandong Province "The Inheritance and Development of Architecture in her Perspective"(zx2015018) .

\section{References}

[1] Koukoumidis, Emmanouil:SignalGuru: Leveraging mobile phones for collaborative traffic signal schedule advisory (2011)

[2] Manning, Kayla:Temporal playscape design within an existing landscape dynamic (2011)

[3] Brunetto, Leah B:Infinite urban landscapes : a journey through Cambridge, Massachusetts (2012)

[4] Pink,Sarah,Urban social movements and small places,City:analysis of urban

trends,culture, theory,policy, action (2011)

[5] Information on http://www.cnki.com.cn/ 